\title{
Efficacy and safety of recanalization with transseptal needle for chronic total occlusion of the brachiocephalic vein in hemodialysis patients
}

\author{
Xi Yin ${ }^{1,2 *}$, Xi Shen ${ }^{1 *}$, Zhongxin Zhou ${ }^{3}$, Qin Chen ${ }^{1}$, Li Zhou ${ }^{1}$, Tianlei Cui ${ }^{1}$ \\ ${ }^{1}$ Department of Nephrology, West China Hospital, Sichuan University, Chengdu, China; ${ }^{2}$ The Second People's Hospital of Panzhihua, Panzhihua, \\ China; ${ }^{3}$ Department of Vascular Surgery, The Third Affiliated Hospital of Southern Medical University, Guangzhou, China \\ Contributions: (I) Conception and design: T Cui; (II) Administrative support: T Cui; (III) Provision of study materials or patients: T Cui; (IV) \\ Collection and assembly of data: X Yin, X Shen, Z Zhou, Q Chen; (V) Data analysis and interpretation: X Yin, X Shen, L Zhou; (VI) Manuscript \\ writing: All authors; (VII) Final approval of manuscript: All authors. \\ "These authors contributed equally to this work. \\ Correspondence to: Tianlei Cui, MD. Department of Nephrology, West China Hospital, Sichuan University, 37 Guoxue Alley, Wuhou District, \\ Chengdu, China. Email: tianleicui@163.com.
}

Background: Whilst there are effective techniques for the recanalization of central venous occlusions, recanalization of chronic total occlusions remains particularly challenging. This study aims to evaluate the safety and efficacy of recanalization using a transseptal needle in chronic total occlusions of the right brachiocephalic vein (RBV) in long-term hemodialysis patients.

Methods: In this single-center, retrospective study, maintenance hemodialysis patients with chronic total occlusion of the RBV were enrolled between February 2017 to March 2019 from West China Hospital of Sichuan University. Refractory lesions were defined as complete vascular occlusions with failed recanalization using conventional techniques. Occlusions were approached using a transseptal needle to penetrate which offers an alternative strategy with firm support force. Patient data, treatment outcomes and patency rates were collected and analyzed to assess the safety and efficacy of the technique.

Results: A total of 16 eligible patients were analyzed. The operation was successful in 13 of the 16 patients and the success rate was $81.25 \%$ (13/16). Twelve patients underwent percutaneous balloon dilatation and stent implantation after sharp recanalization, whilst one patient underwent balloon dilatation only. We achieved procedural success in 13 patients without surgical complications, and all of the patients were discharged in a stable condition. The primary patency rates at 3, 6 and 12 months after surgery were $100 \%, 84.6 \%$ and $69.2 \%$, respectively. The primary assisted patency rates were $100 \%, 84.6 \%$ and $76.9 \%$, respectively, and the secondary patency rates were $100 \%, 84.6 \%$ and $76.9 \%$, respectively.

Conclusions: This study demonstrates that recanalization of chronic total occlusions to the RBV using a transseptal needle is a safe and effective method after traditional guide wire and catheter techniques fail. It was also found that additional techniques are needed for recanalization in patients with RBV occlusion combined with proximal stenosis or occlusion of the right subclavian vein.

Keywords: Transseptal needle; right brachiocephalic vein (RBV); chronic total occlusion; hemodialysis; percutaneous transluminal angioplasty (PTA); percutaneous transluminal angioplasty and stenting (PTAS)

Submitted Jun 19, 2020. Accepted for publication Aug 27, 2020.

doi: $10.21037 / \mathrm{atm}-20-5369$

View this article at: http://dx.doi.org/10.21037/atm-20-5369 


\section{Introduction}

Central venous occlusion is the most common complication of patients undergoing regular hemodialysis treatment. It is caused by mechanical damage to vessel walls from catheter insertion, as well as high flow rate of a preexisting arteriovenous fistula, resulting in intimal hyperplasia, fibrosis, endothelial cell dysfunction, and narrowing and occlusion of blood vessels (1-3). This can reduce the efficacy of hemodialysis by causing ipsilateral limb swelling and vascular access dysfunction, ultimately increasing allcause mortality (4). In day-to-day clinical practice, effective and safe treatment approaches are gradually developing, involving surgical and endovascular intervention. Although effective, invasive surgery can be difficult for patients with poor health such as long-term hemodialysis patients (5-7). Recently, endovascular intervention has become the first-line treatment for central venous occlusion in patients on hemodialysis $(8,9)$. However, recanalization in patients with long-segment occlusions (more than $2 \mathrm{~cm}$ ) and/or angular occlusions still remains a challenge (10). Methods for recanalization are traditional recanalization including regular guide wire and 21-guage needle or the sheath in combination with the dilatator or the stiff end of hydrophilic wire and sharp recanalization including PTCD puncture needle, TIPS needle and transseptal needle. Transseptal needle is used in transseptal puncture which is a critical step in achieving left atrial access for a variety of cardiac procedures. It is thinner and harder than other puncture needles and guide wires, as well as its small curved tips, making it a potentially superior choice for recanalization in this population of patients. Figure 1 showed the transseptal needle. Aside from a case report utilizing transseptal needle to recanalize total occluded segment (4 $\mathrm{mm}$ ), transseptal needle have not been studied for successful use in long-segment occlusions (more than $2 \mathrm{~cm}$ ) and/or angular occlusions (11). Therefore, the purpose of this study was to evaluate the efficacy and safety of sharp recanalization using a transseptal needle after failure using standard guide wire and catheters in hemodialysis patients with chronic total occlusion of the right brachiocephalic vein $(\mathrm{RBV})$.

We present the following article in accordance with the STROBE reporting checklist (available at http://dx.doi. org/10.21037/atm-20-5369).

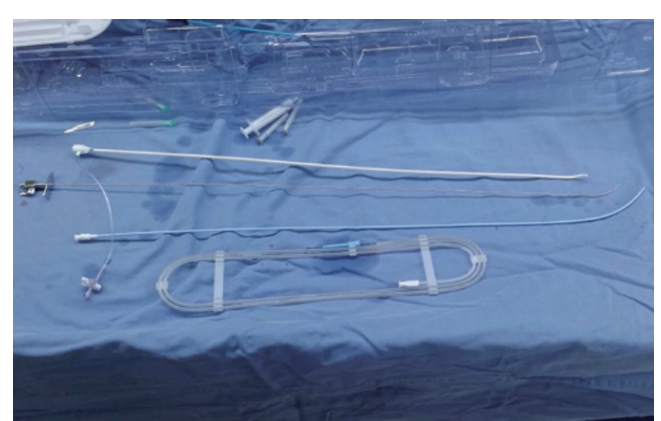

Figure 1 The Transseptal Needle Set consists of a transseptal needle, obturator, and transseptal guiding introducer.

\section{Methods}

\section{Study population}

We retrospectively enrolled hemodialysis patients with chronic total occlusion of the brachiocephalic vein between February 2017 to May 2019 from West China Hospital of Sichuan University. Patients who underwent conventional recanalization were included in this study. The inclusion criteria were as follows: patients with occlusion of the RBV leading to dysfunction of the arteriovenous internal fistula; patients were male or non-pregnant females $>14$ years old; patients with confirmed complete occlusion of the RBV through digital subtraction angiography (DSA) or threedimensional (3D) reconstruction of chest CT. Patients with a history of tumors and/or had an intravascular device such as a pacemaker were excluded. We also excluded patients with an allergy to the contrast medium, swelling of limbs caused by skin soft tissue infection, and coagulation dysfunction. Patient demographic information and treatment outcomes were recorded. All procedures performed in this study involving human participants were in accordance with the Declaration of Helsinki (as revised in 2013). This study was approved by the ethics committee of West China Hospital of Sichuan University (2017204). Because of the retrospective nature of the research, the requirement for informed consent was waived.

\section{Surgical technique}

Thoracic contrast-enhanced CT scans and 3D virtual model reconstructions were performed prior to the procedure 
for all patients. All procedures were performed with local analgesia. Under DSA guidance with the patient in a supine position, 9F, 5F (or $6 \mathrm{~F}$ ) vascular sheaths were inserted at the femoral and forearm (or upper extremity fistula) sites respectively. A contrast medium was administered to delineate the anatomy of the adjacent vessels and occlusion to identify the degree and the accurate length of the occluded segment. Multiple approaches were made from both sides to penetrate the occlusion with the soft or hard end of a 0.035 inch $(1$ inch $\approx 0.025 \mathrm{~m}$ ) hydrophilic guide wire (Terumo, Tokyo, Japan). If this failed, the upper guide wire was advanced to the distal end of the occlusion as a target. Aided by two orthogonal projections in the anteriorposterior and lateral view under DSA, a transseptal needle BRK (St. Jude Medical; St. Paul, MN, USA) was used to retrogradely cross through the femoral vein. Confirmation was obtained that the wires crossed in an appropriate route consistent with the occlusion segment. Next, balloons of different sizes were sequentially passed over the guide wire to produce graded dilations (Cordis Corporation, Milpitas, CA, USA). After dilation, contrast medium was administered in order to rule out extravasation and verify the patency of the occlusive segment. If necessary, stent implantation was performed. Figure 2 shows the surgical procedure. Technical success was defined as successful recanalization followed by balloon angioplasty and stent implantation without major complications. All procedures were performed by the same experienced interventional nephrologists. At follow-up, the primary and secondary patency rates, along with primary assisted patency rates at 3 , 6 and 12 months were recorded.

\section{Statistical analysis}

SPSS 26.0 (Chicago, IL, USA) was used for statistical analysis. The continuous variables conforming to a normal distribution were expressed as mean \pm standard deviation. And the qualitative data were expressed as frequencies or percentages (\%). Patency rates were assessed using the Kaplan-Meier method.

\section{Results}

\section{Patient characteristics}

A total of 16 patients were included in this study (Table 1), including 11 males (71.4\%) and 5 females (28.6\%). The average age was $56.4 \pm 17.2$ years. The patients had received hemodialysis for an average of $54.0 \pm 33.4$ months. The average occlusion length in the RBV was $28.7 \pm 5.1 \mathrm{~mm}$. There were 9 patients with occlusion of the RBV only, whilst other patients had several other comorbid lesions including occlusions to the right internal jugular vein, right subclavian vein and the superior vena cava, or stenoses. A summary of patient demographics is presented in Table 1 .

\section{Surgical success}

Sharp recanalization of the brachiocephalic vein was performed successfully in 13 patients, with a success rate of $81.25 \%(13 / 16)$ and without any serious complication. Only 1 patient underwent percutaneous balloon angioplasty exclusively, whilst 12 patients underwent both percutaneous balloon angioplasty and stent implantation after sharp recanalization. Two of the remaining patients who suffered from occlusion of the RBV combined with proximal right subclavian vein, accepted percutaneous superior vena cava puncture straightly to placement tunneled cuffed catheters with excellent success after failed in sharp recanalization. In one of the patients, a 14 years old female with occlusion of the RBV and superior vena cava, recanalization was not performed as her family did not consent to another operation. Details of occlusion length and treatment outcomes are presented in Table 2.

\section{Clinical outcomes}

The median follow-up was 12 (range, 3-24) months. The primary patency rates at 3, 6 , and 12 months were $100 \%$, $84.6 \%$, and $69.2 \%$, respectively. Primary assisted patency rates were $100 \%, 84.6 \%$, and $76.9 \%$, respectively, whilst the secondary patency rates were $100 \%, 84.6 \%$, and $76.9 \%$, respectively (Figure 3). Two patients were lost at follow-up, and 3 patients developed stenosis, occlusion and thrombosis at follow-up. At 9-month follow-up, 1 patient had stenosis of the right basilic vein, stenosis with thrombosis at the entrance of right subclavian vein stent, and stenosis of the right innominate vein with thrombosis. At 14 months postsurgery, severe stenosis occurred in the proximal part of the right innominate vein and the distal part of the subclavian vein, with stent thrombosis. In the second patient, the right subclavian vein was occluded 8 months post-surgery, losing the access point. At 13 months post-surgery, stenosis of the right cephalic arch was accompanied by thrombosis. At 17 months post-surgery, there were multiple stenoses in 

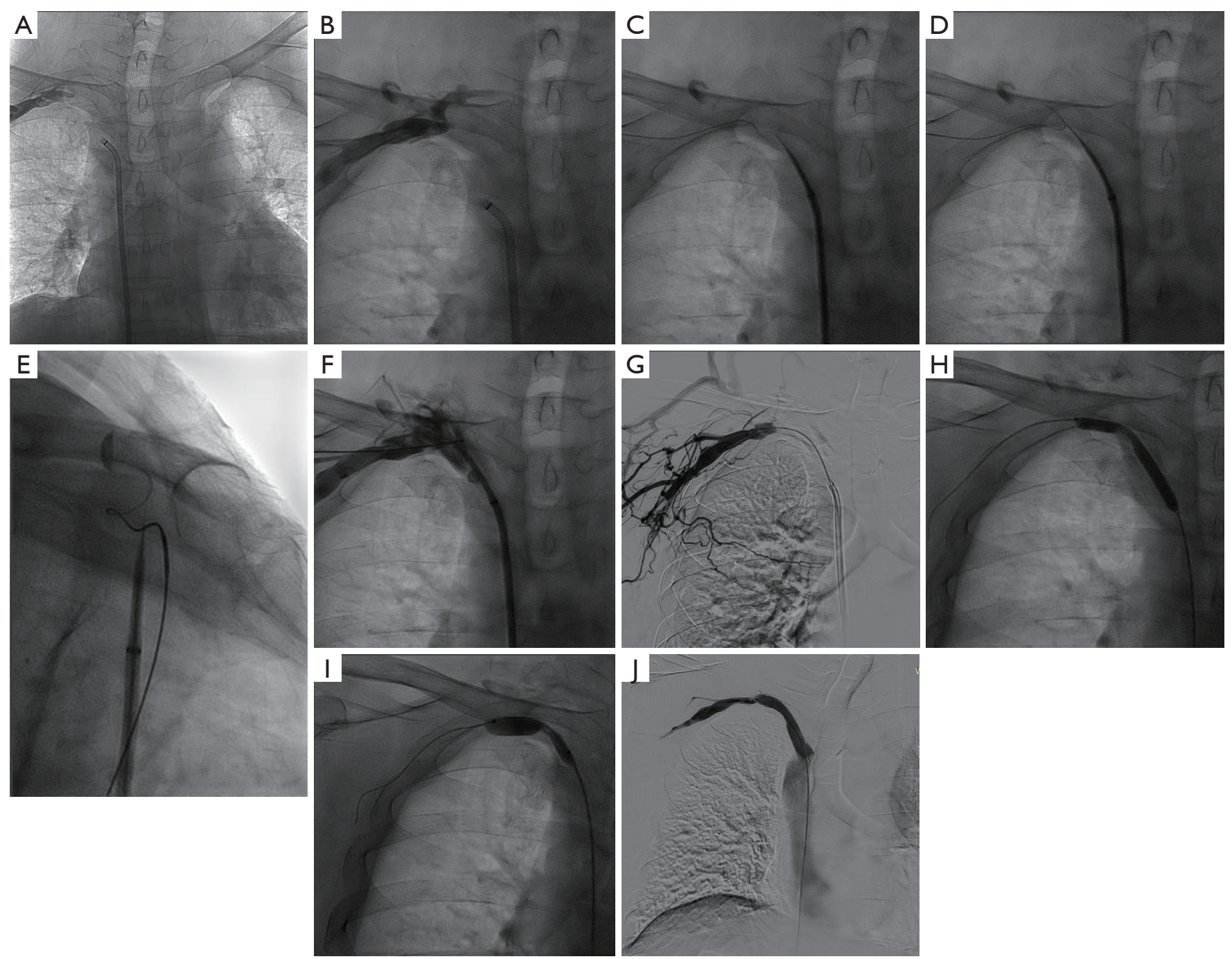

Figure 2 Surgical procedure for the sharp recanalization of the right brachiocephalic vein. (A) Contrast medium was injected into the arteriovenous fistula of the right upper limb to show the occluded segment at the distal end of the right brachiocephalic vein, with the sheath of the transseptal needle below; (B) the guide wire reached the subclavian vein, located and performed angiography; (C) attempt to cross along the direction of the guide wire using the transseptal needle; (D) the tip of the needle meeting the guide wire (anteroposterior X-ray); (E) the tip of the transseptal needle also converges with the positioning of the guide wire. Both the anteroposterior and lateral positions are well aligned, indicating that the puncture is successful (lateral X-ray); (F) the subclavian vein can be clearly visualized when the contrast medium is injected into the sheath of the transseptal needle through the femoral vein; $(\mathrm{G})$ a venogram after puncturing showed complete occlusion of the RBV; $(\mathrm{H})$ the most serious occlusions were found by $6 \mathrm{~mm}$ balloon dilation; (I) a $10 \mathrm{~mm}$ balloon was used for dilation; (J) $10 \mathrm{~mm} \times 6 \mathrm{~cm}$ covered stent (fluency, Bard) was implanted.

the right axillary vein, subclavian vein and the stent. In the third patient, stenoses occurred in the right axillary vein, subclavian vein and brachiocephalic vein 14 months postsurgery. These three patients were treated with balloon dilation and stent implantation for stenosis and occlusion, restoring blood flow.

\section{Discussion}

In this case series, recanalization with a transseptal needle was performed in 16 patients who had chronic total occlusion in the RBV. The success rate of this technique in the present study was higher than previous reports, reaching $81.25 \%$. The surgery was able to successfully 
penetrate the occlusion of the RBV, overcoming the failures of conventional recanalization methods.

Central vein occlusion is a serious problem facing long-

Table 1 Patient demographics and baseline characteristics

\begin{tabular}{lc}
\hline Demographics & Patients $(\mathrm{n}=16)$ \\
\hline Age [years] & $56.4 \pm 17.2[14-77]$ \\
Sex & $11(71.4)$ \\
Male & $5(28.6)$ \\
Female & $54 \pm 33.4[12-120]$ \\
Dialysis duration & \\
Primary disease & $5(31.3)$ \\
Hypertension & $3(18.8)$ \\
Diabetes & $2(12.5)$ \\
Arthrolithiasis & $1(6.25)$ \\
Polycystic kidney & $1(6.25)$ \\
Allergic purpura & $7(43.8)$ \\
Unknown &
\end{tabular}

Data is expressed as mean \pm SD (range) or $n(\%)$. term hemodialysis patients, often exhausting all alternative sites of access, leading to reduced long-term survival rates. It has been reported that the prevalence of central venous occlusion in these patients varies considerably, ranging from $4.3 \%$ to $41 \%(12,13)$. For these patients, even in the absence of central vein catheterization, using the arteriovenous fistula as an access site for hemodialysis may also eventually lead to narrowing or occlusion of the central vein. The mechanisms of central vein occlusion involve extrinsic and intrinsic factors. The former are attributed to compression by adjacent anatomical structures, and the latter is mainly attributed to smooth muscle hyperplasia, mural thrombosis or fibrosis due to intravascular device insertion and high flow rate of the arteriovenous fistula (14). The RBV, also known as the innominate vein, originates from the merging of the right subclavian vein and right internal jugular vein, and converges with the RBV to form the superior vena cava. The length is usually 2 to $3 \mathrm{~cm}$ (15). Occlusion of the RBV could decrease the ipsilateral upper extremity hemodialysis access flow rates and lead to upper limb swelling (16).

Intravascular interventional therapy, namely percutaneous balloon angioplasty, is a standard treatment for central vein

Table 2 Occlusion characteristics and treatment outcomes

\begin{tabular}{lccccc}
\hline Patient & Occluded vein & Occlusion length $(\mathrm{mm})$ & Balloon $(\mathrm{mm})$ & Stent $(\mathrm{mm})$ & \multicolumn{2}{c}{ Successful } \\
\hline 1 & RBV, RIJV & 37 & $6,10,12$ & 12 & Yes \\
2 & RBV & 23 & 8 & 8 & Yes \\
3 & RBV & 39 & $6,8,10,12$ & - & Yes \\
4 & RBV & 28 & $4,8,10$ & 12 & Yes \\
5 & RBV & 23 & $4,8,10$ & 10 & Yes \\
6 & RBV & 29 & 10,12 & 12 & Yes \\
7 & RBV, RSV & 25 & $6,8,10$ & 10 & Yes \\
8 & RBV & 36 & 6,10 & 12 & Yes \\
9 & RBV, RIJV & 24 & $4,6,8$ & 12 & Yes \\
10 & RBV, RSV & 33 & 10,12 & 6,12 & Yes \\
11 & RBV & 24 & $4,5,6$ & 10 & Yes \\
12 & RBV & 25 & 8,10 & 11 & Yes \\
13 & RBV & 28 & 8,12 & - & Yes \\
14 & RBV, SVC & 26 & - & - & No \\
15 & RBV, RSV & 28 & - & - & No \\
16 & RBV, RSV & 31 & - & No \\
\hline
\end{tabular}

RBV, right brachiocephalic vein; RSV, right subclavian vein; RIJV, right internal jugular vein; SVC, superior vena cava. 


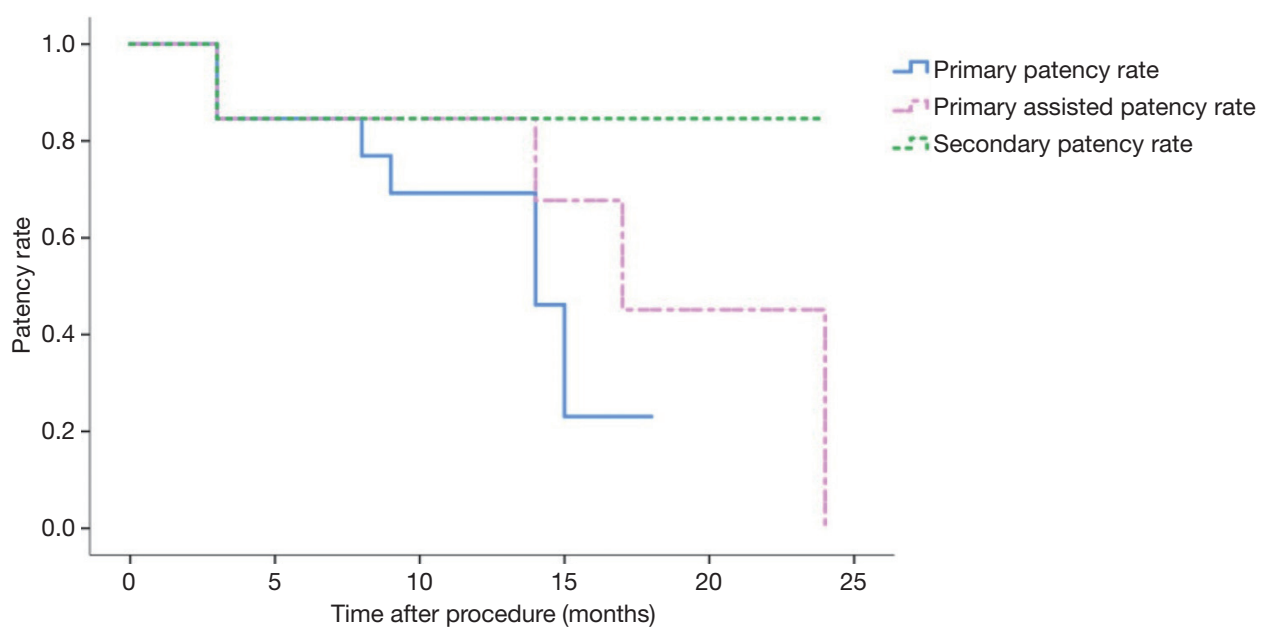

Figure 3 Kaplan-Meier plot depicting the primary, primary assisted and secondary patency rates of the operation. Primary patency of the central vein in long-term is not good, but repeat interventions offer durable outcomes which prolong the primary assisted patency and secondary patency.

occlusion. This technique utilizes the anterior or posterior end of a guide wire, coaxially supporting the catheter and vessel sheath, whilst delivering continuous balloon dilation followed by stent implantation (5). Although primary patency rates of percutaneous balloon angioplasty and stents vary, this technique still has limitations, and secondary patency can be obtained with repeated surgery. In a study of 70 patients with hemodialysis-related central venous stenosis, Quaretti et al. (17) reported a primary patency rate of $58 \%$ at 12 months post-percutaneous balloon angioplasty. Similarly, in a study of 74 patients with symptomatic central vein occlusion, Saleh et al. (18) reported a primary patency rate of $51.7 \% \pm 7.4 \%$ for the percutaneous balloon angioplasty group, and $70.6 \% \pm 11.1 \%$ in the stent group. In the previous study, covered stent grafts resulted in a primary patency rate of $91.7 \%$ at 12 months post-surgery, with an excellent secondary patency rate of $95.8 \%$ when used for central venous stenosis in hemodialysis patients (19). The primary patency rate in our study was higher, but the long-term patency rate was still not encouraging. As a result, repeated interventions may be required to maintain patency. Surgery success rates and long-term patency rates are influenced by several factors, including comorbidities, severity of lesions, fistula flow, types of instruments used and interventional experience of the surgeons.

The first step in percutaneous balloon angioplasty is feeding the guide wire through the occlusion intraluminally. This process is called "crossing" and is a key step towards the success of the surgery. However, there are several studies reporting difficulties in crossing the total occlusion segment during conventional revascularization. The first clinicians to describe sharp recanalization were Gupta et al. (20) and Farrell et al. (21) in 1998 and 1999. A hollow-core puncture needle was used to pass through the occluded segment with a target balloon, then a conventional guide wire and catheter are used. The 21-gauge micro-puncture kit gradually replaced the first 18-gauge puncture needle (22). In 2005, Honnef et al. (23) reported a successful case which traversed a $2 \mathrm{~cm}$ occlusion of the RBV with transjugular intrahepatic portosystemic shunt (TIPS) set directed by the right brachiocephalic stent. A Rosch-Uchida needle was previously used in 33 patients with a subclavian vein or brachiocephalic vein occlusion with a revascularization success rate of $93.9 \%$. The primary patency rates at 3-, 6-, and 12 -month were $43.6 \%, 24 \%$, and $8 \%$, respectively, and the secondary patency rates were $77.4 \%, 68.8 \%$ and $55.9 \%$, respectively (24). The patency rates in our study compared with the Rosch-Uchida needle technique were significantly higher. Several studies using radiofrequency wires (RF), which use radiofrequency energy to generate heat and vaporize cells in contact with the wire tip during sharp recanalization, have been reported in chronic central venous occlusion patients (25-28). However, this technique also penetrates the adjacent soft tissue, potentially resulting in fatal complications. Subsequently, in a study by Arabi et al. (29), transseptal needles were used for sharp central venous recanalization in 6 patients.

It is easier in recanalization of partly $\mathrm{RBV}$ occlusion, and 
there were many methods to make it come true, including mother-child technique, extra stiff guidewire, through-andthrough wire technique, segmented sharp recanalization strategy, as well as using transseptal needle (30-33). Nevertheless, there are limited data and explicit procedure on sharp recanalization of complete RBV occlusions before, our study focused on total RBV occlusion and didn't include partly one. We performed recanalization using a transseptal needle in 16 patients who all had chronic total occlusion of the RBV, confirming that the technique is safe and effective in a larger sample size. Malik et al. (11) reported the use of a transseptal needle to cross the RBV through the femoral vein, with an occlusion degree of $4 \mathrm{~mm}$. The local intima hyperplasia and dense fibrosis of the dysfunctional vessels form fibrous cord tissue. The guide wire or puncture needle is able to easily pass through the dense tissue, breaking through the weak site in the normal position to the outside of the vascular cavity, resulting in rupture and bleeding $(8,34)$. The main advantages of the transseptal puncture needle over other puncture needles include that the needle can adjust its curvature according to the angiographic path, controlling degree and direction. In addition, the diameter of the transeeptal needle is $21 \mathrm{G}$, but it is $16 \mathrm{G}$ of Rosch-Uchida needle and 20G of TIPS, so that the severity of the perforation complications is reduced $(23,24,29)$. For long segment (length $>2 \mathrm{~cm}$ ), it is advisable to use the transseptal puncture needle kit to perform the recanalization operation. In the present study, we found that recanalization of the RBV is easier than recanalization of angular (such as subclavian vein combined with brachiocephalic vein) occlusions. The brachiocephalic vein and the superior vena cava are adjacent to the pericardium, lungs and other vital organs. Serious complications such as pneumothorax, hemothorax, hemopneumothorax, pericardial tamponade or major artery injury may occur if the needle direction deviates. It is difficult to anterogradely puncture chronic vascular occlusions, particularly superior vena cava occlusions. Combining anterograde and reverse puncture from upper limb veins, neck vein and femoral vein is a more effective method. Apart from the initial catheter, a support catheter or vascular sheath is also required in order to provide sufficient stiffness and orientation. Most of the occlusion can be crossed through different angles and bidirectional exploration under X-ray fluoroscopy. After that, percutaneous balloon angioplasty is necessary. Because of the chronic fibrosis characteristics of the occlusion, a balloon dilation is carried out step-by-step until it restores blood flow (35).
In summary, we describe a successful technique using a transseptal puncture needle in the treatment of chronic total occlusion of the RBV. This technique was found to be safe and feasible when carried out by a multidisciplinary team of experienced physicians.

\section{Acknowledgments}

Funding: None.

\section{Footnote}

Reporting Checklist: The authors have completed the STROBE reporting checklist. Available at http://dx.doi. org/10.21037/atm-20-5369

Data Sharing Statement: Available at http://dx.doi. org/10.21037/atm-20-5369

Conflicts of Interest: All authors have completed the ICMJE uniform disclosure form (available at http://dx.doi. org/10.21037/atm-20-5369). The authors have no conflicts of interest to declare.

Ethical Statement: The authors are accountable for all aspects of the work in ensuring that questions related to the accuracy or integrity of any part of the work are appropriately investigated and resolved. All procedures performed in this study involving human participants were in accordance with the Declaration of Helsinki (as revised in 2013). This study was approved by the ethics committee of West China Hospital of Sichuan University (2017204). Because of the retrospective nature of the research, the requirement for informed consent was waived.

Open Access Statement: This is an Open Access article distributed in accordance with the Creative Commons Attribution-NonCommercial-NoDerivs 4.0 International License (CC BY-NC-ND 4.0), which permits the noncommercial replication and distribution of the article with the strict proviso that no changes or edits are made and the original work is properly cited (including links to both the formal publication through the relevant DOI and the license). See: https://creativecommons.org/licenses/by-nc-nd/4.0/.

\section{References}

1. Yew KL, Anderson S, Farah R, et al. Bilateral central 
vein stenosis in a dialysis patient with a pacemaker. Asian Cardiovasc Thorac Ann 2014;22:979-80.

2. Poinen K, Quinn RR, Clarke A, et al. Complications From Tunneled Hemodialysis Catheters: A Canadian Observational Cohort Study. Am J Kidney Dis 2019;73:467-75.

3. Krishna VN, Eason JB, Allon M. Central Venous Occlusion in the Hemodialysis Patient. Am J Kidney Dis 2016;68:803-7.

4. Zhao Y, Yang L, Mai H, et al. Long-segment central venous occlusion in a hemodialysis patient treated by segmented sharp recanalization strategy: A case report. Medicine 2019;98:e15208.

5. Dammers R, de Haan MW, Planken NR, et al. Central vein obstruction in hemodialysis patients: results of radiological and surgical intervention. Eur J Vasc Endovasc Surg 2003;26:317-21.

6. Ayarragaray JE. Surgical treatment of hemodialysis-related central venous stenosis or occlusion: another option to maintain vascular access. J Vasc Surg 2003;37:1043-6.

7. Miyayama S, Minami T, Kozaka K, et al. Small needle puncture of a central venous occlusion in a hemodialysis patient that could not be traversed by a conventional technique. Cardiovasc Interv Ther 2014;29:261-5.

8. Kundu S. Central venous obstruction management. Semin Intervent Radiol 2009;26:115-21.

9. Tordoir JH, Bode AS, Peppelenbosch N, et al. Surgical or endovascular repair of thrombosed dialysis vascular access: is there any evidence? J Vasc Surg 2009;50:953-6.

10. Malgor RD, Wood EA, Gasparis AP, et al. Endovascular strategy for recanalization of long-segment central vein occlusion with concomitant arteriovenous fistula creation. Ann Vasc Surg 2012;26:1012.e17-20.

11. Malik AK, Bhalla N, Goel A, et al. Percutaneous reconstruction of chronic total occlusion of brachiocephalic vein using transseptal needle in dialysis-dependent patient. Cardiovasc Interv Ther 2016;31:136-9.

12. MacRae JM, Ahmed A, Johnson N, et al. Central vein stenosis: a common problem in patients on hemodialysis. ASAIO J 2005;51:77-81.

13. Adwaney A, Lim C, Blakey S, et al. Central Venous Stenosis, Access Outcome and Survival in Patients undergoing Maintenance Hemodialysis. Clin J Am Soc Nephrol 2019;14:378-84.

14. Dolmatch BL, Gurley JC, Baskin KM, et al. Society of Interventional Radiology Reporting Standards for Thoracic Central Vein Obstruction: Endorsed by the American Society of Diagnostic and Interventional
Nephrology (ASDIN), British Society of Interventional Radiology (BSIR), Canadian Interventional Radiology Association (CIRA), Heart Rhythm Society (HRS), Indian Society of Vascular and Interventional Radiology (ISVIR), Vascular Access Society of the Americas (VASA), and Vascular Access Society of Britain and Ireland (VASBI). J Vasc Interv Radiol 2018;29:454-60.e3.

15. Woodhouse P, Waheed A, Bordoni B. Anatomy, Thorax, Brachiocephalic (Innominate) Veins. In: StatPearls. Treasure Island (FL): StatPearls Publishing; June 29, 2019.

16. Modabber M, Kundu S. Central venous disease in hemodialysis patients: an update. Cardiovasc Intervent Radiol 2013;36:898-903.

17. Quaretti P, Galli F, Moramarco LP, et al. Stent Grafts Provided Superior Primary Patency for Central Venous Stenosis Treatment in Comparison with Angioplasty and Bare Metal Stent: A Retrospective Single Center Study on 70 Hemodialysis Patients. Vasc Endovascular Surg 2016;50:221-30.

18. Saleh M, Ali H, Elbadawy A, et al. Balloon angioplasty with selective stenting strategy in treatment of hemodialysis related central vein occlusive lesions. Int Angiol 2017;36:462-6.

19. Boutrous ML, Alvarez AC, Okoye OT, et al. Stent-Graft Length Is Associated with Decreased Patency in Treatment of Central Venous Stenosis in Hemodialysis Patients. Ann Vasc Surg 2019;59:225-30.

20. Gupta H, Murphy TP, Soares GM. Use of a puncture needle for recanalization of an occluded right subclavian vein. Cardiovasc Intervent Radiol 1998;21:508-11.

21. Farrell T, Lang EV, Barnhart W. Sharp recanalization of central venous occlusions. J Vasc Interv Radiol 1999; 10:149-54.

22. Cohen EI, Beck C, Garcia J, et al. Success Rate and Complications of Sharp Recanalization for Treatment of Central Venous Occlusions. Cardiovasc Intervent Radiol 2018;41:73-9.

23. Honnef D, Wingen M, Günther RW, et al. Sharp central venous recanalization by means of a TIPS needle. Cardiovasc Intervent Radiol 2005;28:673-6.

24. Goo DE, Kim YJ, Choi DL, et al. Use of a roschuchida needle for recanalization of refractory dialysisrelated central vein occlusion. AJR Am J Roentgenol 2010;194:1352-6.

25. Guimaraes M, Schonholz C, Hannegan C, et al. Radiofrequency wire for the recanalization of central vein occlusions that have failed conventional endovascular techniques. J Vasc Interv Radiol 2012;23:1016-21. 
26. Sivananthan G, MacArthur DH, Daly KP, et al. Safety and efficacy of radiofrequency wire recanalization of chronic central venous occlusions. J Vasc Access 2015;16:309-14.

27. Keller EJ, Gupta SA, Bondarev S, et al. SingleCenter Retrospective Review of Radiofrequency Wire Recanalization of Refractory Central Venous Occlusions. J Vasc Interv Radiol 2018;29:1571-7.

28. Majdalany BS, Monfore N, Khaja MS, et al. Radiofrequency Wire Recanalization of Chronically Occluded Venous Stents: A Retrospective, Single-Center Experience in 15 Patients. Cardiovasc Intervent Radiol 2019;42:130-6.

29. Arabi M, Ahmed I, Mat'hami A, et al. Sharp Central Venous Recanalization in Hemodialysis Patients: A SingleInstitution Experience. Cardiovasc Intervent Radiol 2016;39:927-34.

30. Wan Z, Lai Q, Zhou Y, et al. Efficacy and safety of a mother-child technique for recanalization of chronic central venous occlusive disease in hemodialysis patients. J

Cite this article as: Yin X, Shen X, Zhou Z, Chen Q, Zhou L, Cui T. Efficacy and safety of recanalization with transseptal needle for chronic total occlusion of the brachiocephalic vein in hemodialysis patients. Ann Transl Med 2020;8(18):1141. doi: 10.21037/atm-20-5369
Vasc Surg Venous Lymphat Disord. 2020;8(4):558-564.

31. Morinière $\mathrm{P}$, Rodary-Vautier R, Fillioux-Morfaux V, et al. Percutaneous recanalization of occlusion of central and proximal veins in chronic hemodialysis. Technical note. Kidney Int 1997;52:1406-11.

32. Huang Y, Chen B, Tan G, et al. The feasibility and safety of a through-and-through wire technique for central venous occlusion in dialysis patients. BMC Cardiovasc Disord 2016;16:250.

33. Zhao Y, Yang L, Mai H, et al. Long-segment central venous occlusion in a hemodialysis patient treated by segmented sharp recanalization strategy: A case report. Medicine (Baltimore) 2019;98:e15208.

34. Shi YX, Ye M, Liang W, et al. Endovascular treatment of central venous stenosis and obstruction in hemodialysis patients. Chin Med J (Engl) 2013;126:426-30.

35. Vachharajani TJ, Agarwal AK, Asif A. Vascular access of last resort. Kidney Int 2018;93:797-802. 